\title{
Preparation and evaluation of quercetin-loaded lecithin-chitosan nanoparticles for topical delivery
}

This article was published in the following Dove Press journal:

International Journal of Nanomedicine

9 August 2011

Number of times this article has been viewed

\section{Qi Tan' \\ Weidong Liu' ${ }^{1,2}$ \\ Chenyu Guo' \\ Guangxi Zhai'}

'Department of Pharmaceutics, College of Pharmacy, Shandong University, Jinan, ${ }^{2}$ Department of Pharmacy, Linyi People's Hospital Affiliated to Shandong University, Linyi, People's Republic of China
Correspondence: Guangxi Zhai Department of Pharmaceutics, College of Pharmacy, Shandong University, 44 Wenhua Xilu, Jinan 2500 12, People's Republic of China $\mathrm{Tel}+8653188382015$

Email professorgxzhai@yahoo.cn
Background: The purpose of this study was to investigate lecithin-chitosan nanoparticles as a topical delivery system for quercetin.

Methods: Tocopheryl propylene glycol succinate was chosen to be the surfactant for the nanosystem. The mean particle size of the nanoparticles was $95.3 \mathrm{~nm}$, and the entrapment efficiency and drug loading for quercetin were $48.5 \%$ and $2.45 \%$, respectively. Topical delivery in vitro and in vivo of the quercetin-loaded nanoparticles was evaluated using quercetin propylene glycol solution as the control.

Results: Compared with quercetin solution, the quercetin-loaded nanoparticles showed higher permeation ability, and significantly increased accumulation of quercetin in the skin, especially in the epidermis. Microstructure observation of the skin surface after administration indicated that the interaction between ingredients of the nanoparticles and the skin surface markedly changed the morphology of the stratum corneum and disrupted the corneocyte layers, thus facilitating the permeation and accumulation of quercetin in skin.

Conclusion: Lecithin-chitosan nanoparticles are a promising carrier for topical delivery of quercetin.

Keywords: quercetin, tocopheryl polyethylene glycol succinate, lecithin, chitosan, nanoparticles, topical delivery

\section{Introduction}

Skin is the largest organ of the human body and is directly exposed to the environment. Therefore, skin often suffers oxidative stress caused by environmental factors, such as ultraviolet irradiation, ionizing radiation, and toxic chemicals. ${ }^{1}$ Although the skin does provide a certain degree of self-protection, it can be overpowered by reactive oxygen species, sustaining severe damage, such as lipid peroxidation, enzyme inactivation, and DNA breakage. Superoxide radicals and hydrogen peroxide are precursors to reactive oxygen species. ${ }^{2,3}$ Developing effective methods to combat reactive oxygen species has been a subject of intense research. Quercetin, a topical antioxidant, is known to have the ability to delay ultraviolet radiation-mediated oxidant injury and cell death by scavenging oxygen radicals, protecting lipids against peroxidation to terminate the chain-radical reaction, and chelating metal ions to form inert complexes that prevent conversion of superoxide radicals and hydrogen peroxide into hydroxyl radicals. ${ }^{4-8}$ Quercetin has superior antioxidant potency compared with many other well known antioxidant molecules, owing to the optimized number and distinctive positions of the free hydroxyl groups in this molecule. ${ }^{9,10}$ However, insolubility of quercetin in water hinders its permeation into skin, and its protection for skin is limited. Therefore, development of 
novel nanovehicles that are capable of transporting quercetin across the skin to exert its bioactivity in inhibiting ultraviolet B-induced cutaneous oxidative stress and inflammation is of great significance.

Nanoparticles have considerable potential in topical delivery of drugs due to their distinctive properties. Because of their increased surface area, nanoparticles have greater efficiency in enhancing the permeation of drugs into skin than many other vehicles. Several studies have shown that nanosized particles tend to reside in the outer layers of the stratum corneum and epidermis, with negligible penetration into the dermis. ${ }^{11-13}$

Lecithin is a natural lipid mixture of phospholipids and is frequently used for the preparation of various nanosystem delivery vehicles, such as microemulsions, liposomes, micelles, and nanoparticles, and is considered to be a safe and biocompatible excipient. ${ }^{14-19}$ Chitosan, a polycationic polymer comprised mainly of glucosamine units, is a N-deacetylated derivative of chitin. It has been reported that chitosan has antioxidant ${ }^{20,21}$ and anti-inflammatory properties, ${ }^{22,23}$ as well as many other attractive biopharmaceutical properties, including biocompatibility, biodegradability, bioadhesion, penetration enhancement, and antimicrobial activity. ${ }^{24-27}$ This polycationic polymer can interact with negatively charged substances to form a core shell nanostructure which has been proved to be a promising carrier for drugs. ${ }^{18,19,28}$ Chitosan has been used in many topical preparations to enhance drug uptake in nasal, buccal, and intestinal epithelia, and skin. ${ }^{29-33}$ It has also been reported that lecithin-chitosan nanoparticles obtained through the supramolecular self-organizing interaction between negatively charged lecithin and positively charged chitosan can become strongly attached to mammalian skin, which is slightly negatively charged, and offers better coverage for topical delivery of active compounds. ${ }^{17-19,34}$

In this study, quercetin-loaded lecithin-chitosan nanoparticles containing D- $\alpha$-tocopheryl polyethylene glycol 1000 succinate (TPGS) were prepared and optimized, and the physicochemical properties of the nanoparticles were characterized. In vitro and in vivo drug distribution in the epidermis and dermis of mice after topical administration of quercetin-loaded lecithin-chitosan nanoparticles was evaluated, and the effect of lecithin-chitosan nanoparticles on the skin surface of the mice was investigated.

\section{Materials and methods}

\section{Materials}

Quercetin was purchased from Xi'an Senmu Biological Technology Co Ltd (Xi'an, China). Soya lecithin was obtained from Shanghai Taiwei Pharmaceutical Co Ltd (Shanghai, China). Chitosan of purity 95\% (molecular weight $200 \mathrm{kDa}, 85 \%$ deacetylation) was obtained from Haidebei Marine Bioengineering Co Ltd (Jinan, China) and used without further purification. TPGS was supplied by Wuhan Yuancheng Co Ltd (Wuhan, China). Tween80 and propylene glycol were purchased from Yongda Chemical Reagent Co Ltd (Tianjin, China). Ethanol and methanol of high-pressure liquid chromatography (HPLC) grade were purchased from Tianjin Guangcheng Chemical Agent Co Ltd (Tianjin, China). Other chemicals and solvents were of analytical reagent grade.

\section{Preparation of nanoparticles}

Lecithin-chitosan nanoparticles were prepared according to an established method. ${ }^{18}$ Lecithin $5 \%(\mathrm{w} / \mathrm{v})$ was dissolved in a $96 \%$ ethanol solution. Quercetin was then dissolved in the ethanol solution containing lecithin to obtain a weight ratio of lecithin to quercetin of 10:3. The chitosan solutions were prepared by dissolving $10 \mathrm{mg}$ of chitosan in $50 \mathrm{~mL}$ of distilled water with $0.1 \%$ acetic acid. TPGS was subsequently dissolved in the chitosan solution, and then $4 \mathrm{~mL}$ of the aforementioned mixed ethanol solution was injected into $46 \mathrm{~mL}$ of chitosan solution through a plastic needle tubing (internal diameter $0.75 \mathrm{~mm}$, injection rate $2 \mathrm{~mL} /$ minute) under mechanical agitation at $1000 \mathrm{rpm}$. After stirring for half an hour, the $\mathrm{pH}$ of the nanoparticle suspension was adjusted to 4.5 with $0.5 \mathrm{M}$ sodium hydroxide solution. After the resulting suspension was filtered through a filter membrane $(0.8 \mu \mathrm{m})$ to remove the nonincorporated drug, the prepared nanoparticle system was obtained. The lecithin-chitosan ratio was set at $20: 1(\mathrm{w} / \mathrm{w})$ in the nanoparticles.

\section{Physicochemical characterization of nanoparticles}

\section{Particle size and zeta potential}

The mean diameter of the quercetin-loaded nanoparticles was measured by photon correlation spectroscopy using a particle sizer (Zetasizer $3000 \mathrm{HAS}$; Malvern Instruments Ltd, Worcestershire, UK) at a fixed angle of $90^{\circ}$ with a He-Ne laser of $633 \mathrm{~nm}$ at $25^{\circ} \mathrm{C}$. Particle size was evaluated using volume distribution. The zeta potential was analyzed using a microscopic electrophoresis system (DXD-II; Jiangsu Optics Co Ltd, Jiangsu, China) at $25^{\circ} \mathrm{C}$.

\section{Morphology}

The morphology of the quercetin-loaded nanoparticles was observed under transmission electron microscopy 
(JEM-1200EX; JEOL, Tokyo, Japan). One drop of diluted quercetin-loaded nanoparticle suspension was deposited on a film-coated copper grid and stained with one drop of $2 \%$ $(\mathrm{w} / \mathrm{v})$ aqueous solution of phosphotungstic acid, and then allowed to dry for contrast enhancement. The sample was then examined by transmission electron microscopy. ${ }^{35}$

\section{Entrapment efficiency and drug loading}

The prepared nanoparticles were separated from the free drug using a Sephadex G-50 minicolumn centrifugation technique for measurement of entrapment efficiency. ${ }^{36,37}$ Briefly, $0.3 \mathrm{~mL}$ of the prepared nanoparticle suspension was placed in a Sephadex G-50 minicolumn (presaturated with empty nanoparticles prepared using the same composition and methods) and centrifuged at $1000 \mathrm{rpm}$ for 1 minute, and the column was then washed seven times with $0.1 \mathrm{~mL}$ of distilled water. Elutes containing drug-loaded nanoparticles were collected and adjusted to a volume of $25 \mathrm{~mL}$ by ethanol, and the elute-ethanol solution was then vortexed for 3 minutes to break down the nanoparticles and dissolve the quercetin. The mixture was then centrifuged at 12,000 rpm for 10 minutes to precipitate the crystallized salt and chitosan. Thereafter, the amount of entrapped drug in the supernatant liquid was detected using an ultraviolet-visible spectrophotometer (Lambda 15; Perkin-Elmer, Schwerzenbach, Switzerland) at a wavelength of $373 \mathrm{~nm}$. The entrapment efficiency and drug-loading of quercetin in the nanoparticles were calculated according to the following equations: ${ }^{38}$

$$
\begin{aligned}
& \mathrm{EE}=\frac{\mathrm{W}_{\text {entrapped drug }}}{\mathrm{W}_{\text {initial drug }}} \times 100 \% \\
& \mathrm{DL}=\frac{\mathrm{W}_{\text {entrapped drug }}}{\mathrm{W}_{\mathrm{NPs}}} \times 100 \%
\end{aligned}
$$

where $\mathrm{W}_{\text {initial drug }}$ represents the initially added amount of drug and $\mathrm{W}_{\text {entrapped drug }}$ represents the amount of drug entrapped in the nanoparticles. $\mathrm{W}_{\mathrm{NP}}$ represents the total weight of all components in the nanoparticles.

\section{In vitro permeation studies Preparation of skins}

The male Kunming mice $(20 \pm 2 \mathrm{~g})$ used in the permeation studies were purchased from the Experimental Animal Center of Shandong University. The study protocol was approved by the ethics committee of Shandong University. The mice were sacrificed and their dorsal hair was carefully removed with a razor. The skins were obtained from the hairless regions of the mice, and the subcutaneous fat and connective tissue were trimmed. The excised skins were washed and examined to ensure their integrity, and were then stored in a refrigerator at $-20^{\circ} \mathrm{C}$ overnight for later use. ${ }^{39}$

\section{In vitro study in mice}

The in vitro permeation experiment was carried out using Franz diffusion cells with an effective diffusion area of $3.14 \mathrm{~cm}^{2}$. The excised skin was thawed and mounted between the donor and receptor chamber of the Franz diffusion cell, with the stratum corneum side facing upwards. A quercetinloaded nanoparticle suspension $(0.5 \mathrm{~mL})$ or control solution with the same quercetin concentration was applied to the surface of the stratum corneum. The receptor chamber was filled with $20 \mathrm{~mL}$ of phosphate-buffered saline $(\mathrm{pH} 7.2)$ containing $1 \%$ Tween 80 which can increase quercetin solubility to $155.8 \mu \mathrm{g} / \mathrm{mL}$ and keep a sink condition for quercetin penetrated through the skin. The receptor medium was kept at $32^{\circ} \mathrm{C} \pm 0.5^{\circ} \mathrm{C}$ and stirred at $300 \mathrm{rpm}$ throughout the experiment. At designated time intervals (hours 0.5, 1, 2, $4,6,8,10,12$, or 24 following application) $0.5 \mathrm{~mL}$ of receptor solution was taken out and $0.5 \mathrm{~mL}$ of blank medium at the same temperature was added into the receptor compartment to keep the volume constant. Acetic ether $3 \mathrm{~mL}$ was added to the sample solution and vortexed for 3 minutes, and then centrifuged at $4000 \mathrm{rpm}$ for 10 minutes. The supernatant was collected and air-dried in nitrogen, and the solid obtained was redissolved in $250 \mu \mathrm{L}$ of methanol, then the amount of quercetin in the methanol solution was analyzed by HPLC.

At hours 3, 6, 9, and 12 after application of drug-loaded nanoparticles and quercetin propylene glycol solution, the skin surfaces were thoroughly washed with distilled water to remove excess formulation, and were then placed above an aqueous bath $\left(60^{\circ} \mathrm{C}\right)$ for 60 seconds to separate the epidermis and dermis according to the established method. ${ }^{40}$ After that, the epidermis and dermis were cut into pieces and put into a plastic test tube $(10 \mathrm{~mL})$. Physiological saline solution $1 \mathrm{~mL}$ was added to each of them, and after being homogenized, extraction of quercetin from the homogenized skin tissue was performed using the same steps as those for the receptor solution described earlier. The amount of quercetin was analyzed by HPLC.

\section{In vivo study in mice}

All the experimental mice were housed in cages, with access to food and water until use. Twenty-four hours prior to the experiment, the dorsal hair was removed with a razor and the bare skins were washed with physiological solution. 
Quercetin-loaded nanoparticle suspension $0.5 \mathrm{~mL}$ (drug content $0.5 \%, \mathrm{w} / \mathrm{w}$ ) or control solution was applied to the dorsal surface $\left(3.14 \mathrm{~cm}^{2}\right)$. At hours 3, 6, 9, and 12 thereafter, the mice were killed by cervical dislocation, and the treated skin area was dissected. Further steps for skin sample treatment and extraction of quercetin from skin tissues were performed using the same methods as those described earlier. The amount of quercetin in the epidermis and dermis was analyzed by HPLC.

\section{Effect of nanoparticles on surface of skin}

The aim of the experiment was to determine the topical effect of quercetin-loaded nanoparticle suspension on mouse skin using a previously reported method. ${ }^{41}$ The nanoparticle suspension and quercetin propylene glycol solution were applied to the hairless dorsal skin of mice for 24 hours. The mice were then killed by cervical dislocation, and the treated skin area was dissected and collected in 4\% formalin for histopathological studies. About 3-4 $\mu \mathrm{m}$ of paraffin tissue sections were cut and subjected to hematoxylin and eosin staining. Microscopic pictures were taken by Nikon Alphaphot-2 YS2 microscope (Tokyo, Japan).

\section{HPLC analysis of quercetin}

The samples were analyzed using an HPLC system (1200 series; Agilent Technologies, Palo Alto, CA) and an HPLC column $(\mathrm{C} 18,4.6 \times 250 \mathrm{~mm}, 5 \mu \mathrm{m})$. The mobile phase was a mixture of methanol-water at a ratio of 50:50 (v/v) containing 3\% acetic acid. The flow rate was set at $1 \mathrm{~mL} /$ minute and quercetin was detected at $373 \mathrm{~nm}$. Aliquots of $20 \mu \mathrm{L}$ of each sample were injected into the column, and all operations were carried out at ambient temperature. The peak area correlated linearly with the quercetin concentration in the range of $1.5-150 \mu \mathrm{g} / \mathrm{mL}$ and the lowest detection limit at $0.5 \mu \mathrm{g} / \mathrm{mL}$.

\section{Statistical analysis}

Data are shown as means \pm standard deviation $(n=5)$. Statistical data were analyzed by the Student's $t$-test at the level of $P=0.05$.

\section{Results and discussion Preparation of quercetin-loaded nanoparticles}

Injection of an alcohol solution containing lecithin into chitosan aqueous solution is a well established method for the preparation of nanoparticles, and based on previous studies, nanoparticles made from lecithin and chitosan exhibited well rounded shapes and better stability when the weight ratio of lecithin to chitosan was 20:1. ${ }^{19,24}$ This ratio was adopted in the present study. TPGS was chosen for its known ability to increase drug solubility, stabilize nanosystems, ${ }^{42-44}$ and improve drug absorption. ${ }^{45,46}$ The visual observation of prepared quercetin-loaded nanoparticles and crude quercetin in water is shown in Figure 1. Crude quercetin was hardly dissolved in water and precipitated from the suspension in a short time, whereas the quercetin-loaded nanoparticle suspension showed a translucent state with visible opalescence.

Figure 2 shows the structure of quercetin, and Figure 3 shows the possible structure of nanoparticles prepared in this work. According to previous studies, lecithin and chitosan can form perfect round nanoparticles by the electrostatic interaction between the polycationic chitosan and negatively charged lecithin, in which lecithin molecules form the core and chitosan molecules form the hydrophilic shell layer to protect the inner structure. ${ }^{18,19}$ In this study, the hydrophobic groups in the TPGS and lecithin molecules intertwine with each other to form the hydrophobic core of the nanoparticles. Also, the hydrophilic polyethylene glycol chain in the molecular structure of TPGS and the hydrophilic parts of the lecithin molecules might participate in the formation of the hydrated shell layer with chitosan. Because of the good lipophilicity of quercetin and the hydrated structure of the shell layer, quercetin should dissolve or disperse in the hydrophobic core, and be protected by the outer shell layer. However, the exact structure of quercetin-loaded nanoparticles still needs further investigation. The effect of TPGS on entrapment efficiency and drug-loading was investigated, as shown in Table 1. Drug-loading was clearly influenced by the concentration of TPGS and increased by more than five-fold

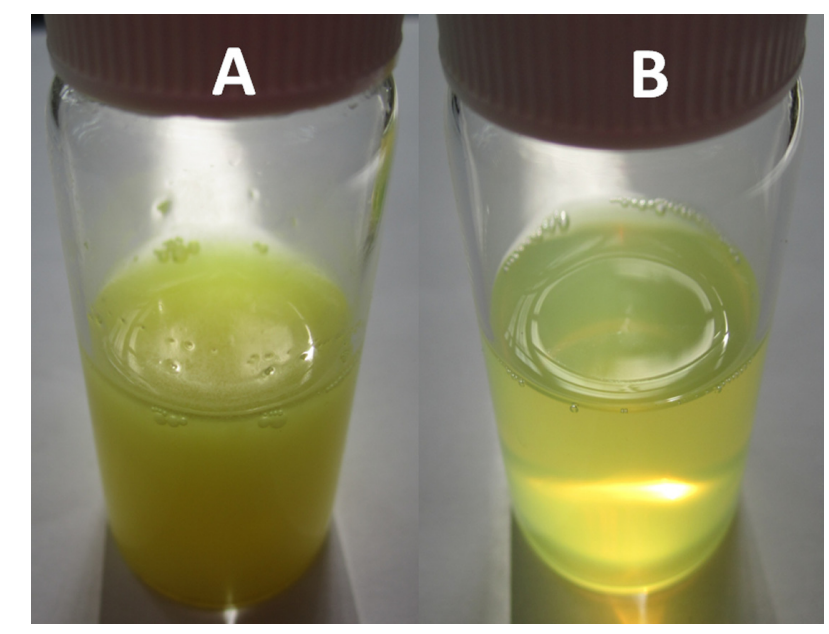

Figure I Visual observation of crude quercetin in water (A) and quercetin-loaded nanoparticle suspension (B). 
<smiles>O=c1c(O)c(-c2ccc(O)c(O)c2)oc2cc(O)cc(O)c12</smiles>

Figure 2 Molecular structure of quercetin.

when the concentration of TPGS changed from $0 \%$ to $2 \%$ $(w / v)$. However, with increasing concentrations of TPGS, drug-loading decreased slightly, indicating that drug-loading reached a maximum level when the TPGS concentration was $2 \%$. Therefore, based on drug-loading, the optimized formulation for the nanoparticles was $0.02 \%$ chitosan, $0.4 \%$ lecithin, and $2 \%$ TPGS.

\section{Characterization of nanoparticles}

The morphology of the nanoparticles was observed under transmission electron microscopy. As shown in Figure 4, the optimized nanoparticles were spherical. The particle size of both the drug-loaded nanoparticles and the blank nanoparticles was $95.3 \mathrm{~nm}$ and $168.0 \mathrm{~nm}$, respectively, and the polydispersity index was 0.441 and 0.331 . The relatively large values for the polydispersity index may be due to the small particle size fraction of formed micelles of TPGS. After quercetin was loaded into particles, the particle size became smaller. A possible reason for this was that the loaded drug enhanced the cohesive force of the hydrophobic interaction and thus caused the decrease in size. ${ }^{47}$

The zeta potential of the drug-loaded nanoparticles and blank nanoparticles was $10.85 \pm 0.05 \mathrm{mV}$ and

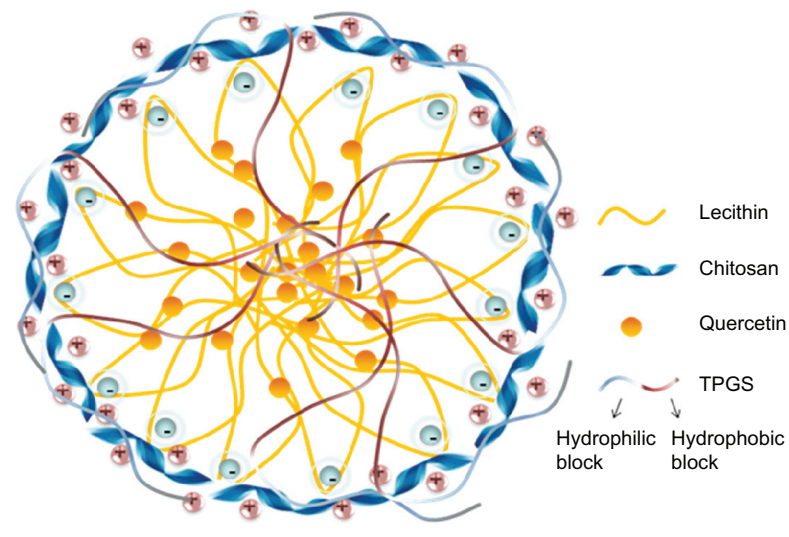

Figure 3 Schematic illustration of possible packing of quercetin-loaded nanoparticles.
Table I Influence of the concentration of TPGS on entrapment efficiency and drug-loading of nanoparticles

\begin{tabular}{lllll}
\hline $\begin{array}{l}\text { TPGS } \\
\text { (\%, w/v) }\end{array}$ & $\begin{array}{l}\text { Lecithin } \\
(\%, w / v)\end{array}$ & $\begin{array}{l}\text { Chitosan } \\
(\%, w / v)\end{array}$ & $\begin{array}{l}\text { EE }^{a} \\
\text { (\%) }\end{array}$ & $\begin{array}{l}\text { DL }^{a} \\
(\%)\end{array}$ \\
\hline 0 & 0.4 & 0.02 & $<10$ & $<0.5$ \\
1 & 0.4 & 0.02 & $45.01 \pm 2.82$ & $1.70 \pm 0.11$ \\
1.5 & 0.4 & 0.02 & $46.30 \pm 1.53$ & $2.21 \pm 0.11$ \\
2 & 0.4 & 0.02 & $48.47 \pm 3.76$ & $2.45 \pm 0.08$ \\
2.5 & 0.4 & 0.02 & $51.07 \pm 1.13$ & $2.32 \pm 0.15$ \\
\hline
\end{tabular}

Note: a Means \pm standard deviation $(n=3)$.

Abbreviations: DL, drug-loading; EE, entrapment efficiency; TPGS, D- $\alpha$-tocopheryl polyethylene glycol 1000 succinate.

$24.78 \pm 0.05 \mathrm{mV}$, respectively. It has been reported that the electrostatic attraction between the most acidic phenolic $\mathrm{OH}$ groups of quercetin and the positively charged particles influences the charge distribution, thus causing a decrease in zeta potential of the whole system..$^{48}$ Although the measured zeta potential was relatively low, the nanoparticle suspension still showed good stability, and there was no notable change in morphology or particle size of the quercetin-loaded nanoparticles during 2 weeks stored at $4^{\circ} \mathrm{C}$. The result was consistent with previous reports of TPGS used as a stabilizer for nanoparticles. ${ }^{49-51}$

\section{In vitro and in vivo percutaneous delivery study}

In the in vitro percutaneous study, quercetin was barely detectable in the receptor cell for 12 hours after administration

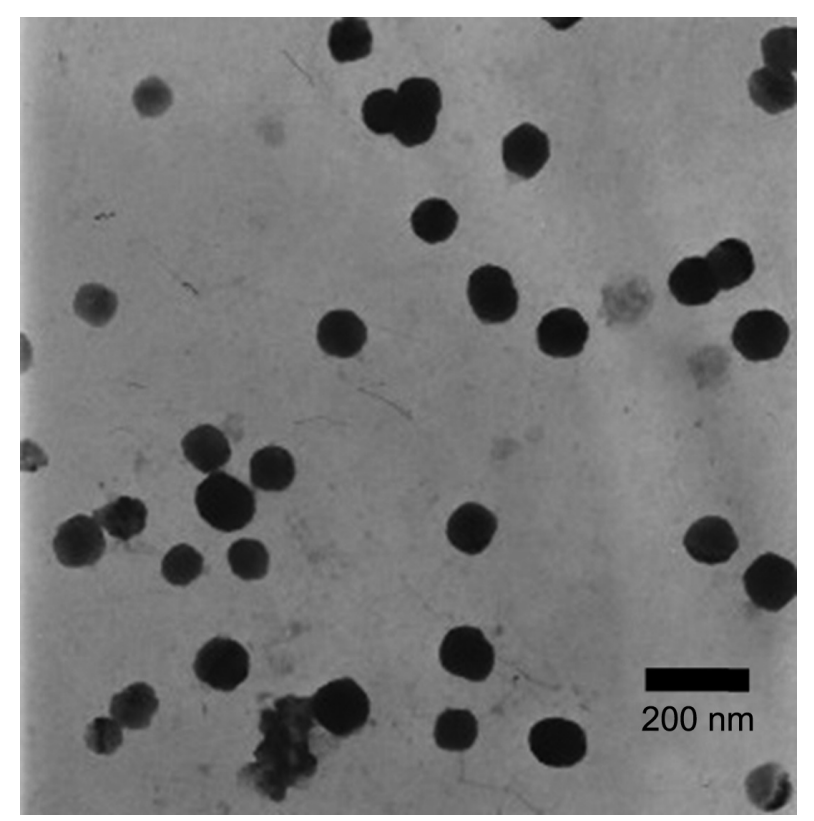

Figure 4 Transmission electron microscopic photograph of quercetin-loaded nanoparticles $(10,000 \times)$. 
of quercetin-loaded nanoparticles or quercetin control solution. The distribution of quercetin in the excised dorsal skins of mice is shown in Figure 5. Compared with the quercetin propylene glycol solution, nanoparticles could markedly enhance the cumulative amounts of quercetin in the dermis and epidermis within 12 hours of application $(P<0.05)$, and the amounts of quercetin in epidermis and dermis were $9.00 \pm 0.40 \mu \mathrm{g}$ and $3.31 \pm 0.51 \mu \mathrm{g}$, respectively, which were 1.45 and 1.32 times these of the control formulation.

In vivo, quercetin extracted from the epidermis and dermis was analyzed by HPLC at every sampling point. Figure 6 shows the amounts of quercetin remaining in the epidermis and dermis at hours 3, 6, 9, and 12 after application of the nanoparticle and control formulations. For the nanoparticles, the amounts of quercetin in the epidermis and dermis were $8.4 \pm 1.53 \mu \mathrm{g}$ and $2.21 \pm 0.01 \mu \mathrm{g}$, respectively, which were 2.3 and 1.2 times those of the control formulation. The permeation behavior of quercetin in vivo was similar to that in vitro $(P<0.05)$.

The statistical difference between the experimental group and the control group in the accumulation of quercetin in the epidermis could be due to the unique characteristics of the prepared nanoparticles, as well as their positive charge and large surface area, ensuring an excellent contact surface between the vehicles and skin over the entire application area and promoting permeation and accumulation of drug in the skin. ${ }^{11,42}$ In addition, the nanoparticles tended to suppress penetration (transdermal delivery) through the skin while enhancing permeation (dermal delivery) of the drug into the upper skin layers. ${ }^{12,13,41,52-54}$ In Figure 6, the amounts of quercetin in the dermis were similar in the control and experimental groups but were clearly different in the epidermis. Because of the highly lipophilic properties of lecithin and quercetin, the carrier cannot transport the drug through the inner more hydrophilic skin layers. In contrast, the alcohol vehicle was relatively more likely to facilitate penetration of the drug into the dermis. ${ }^{55}$ In accordance with the experimental results, under the influence of multiple factors, the gap of drug retention between the experimental group and control group narrowed. Such peculiarity of lecithin-chitosan nanoparticles could improve accumulation of quercetin in the epidermis and avoid its permeation through the skin, which indicates that the lecithin-chitosan nanoparticles are an ideal carrier for the cutaneous delivery of quercetin to exert its antioxidant and anti-inflammatory effects in the skin. Moreover, the structure of lecithin-chitosan nanoparticles could influence the distribution of quercetin in skin tissue. A solid matrix in the core of the nanoparticles could immobilize quercetin molecules and show controlled release for quercetin, which resulted in a low concentration gradient, and thus quercetin stored in nanoparticles could hardly penetrate into the dermis and only distributed in the epidermis, especially in the stratum corneum. ${ }^{19,54}$

On the other hand, TPGS played a very important role in the formulation by disrupting the lipid structure of the stratum corneum to reduce the barrier of skin, and providing better wetting to the skin, which might be essential for bioadhesion and facilitating diffusion of drug through the barrier layer,

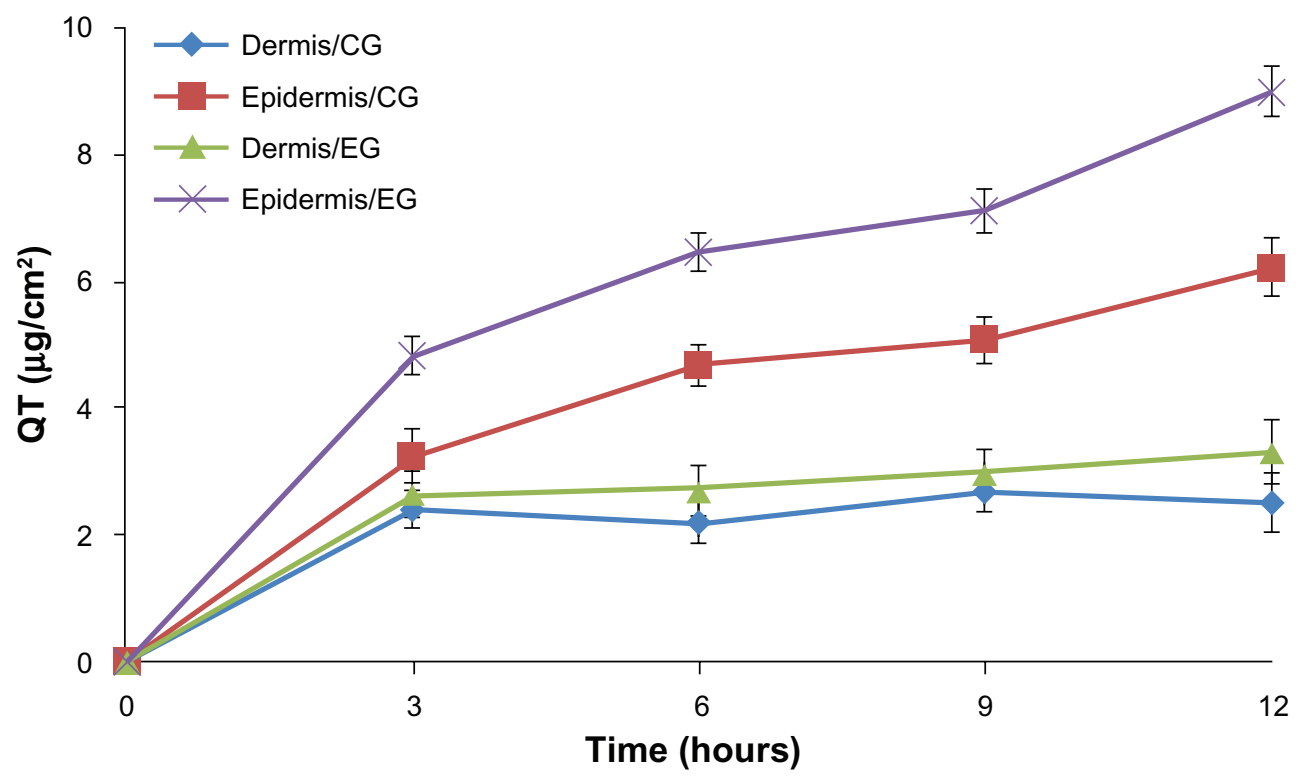

Figure 5 Time course of in vitro skin permeation of quercetin incorporated into nanoparticles or control formulation. Note: Results are represented by means \pm standard deviation $(n=3)$.

Abbreviations: CG, control group; EG, experimental group. 

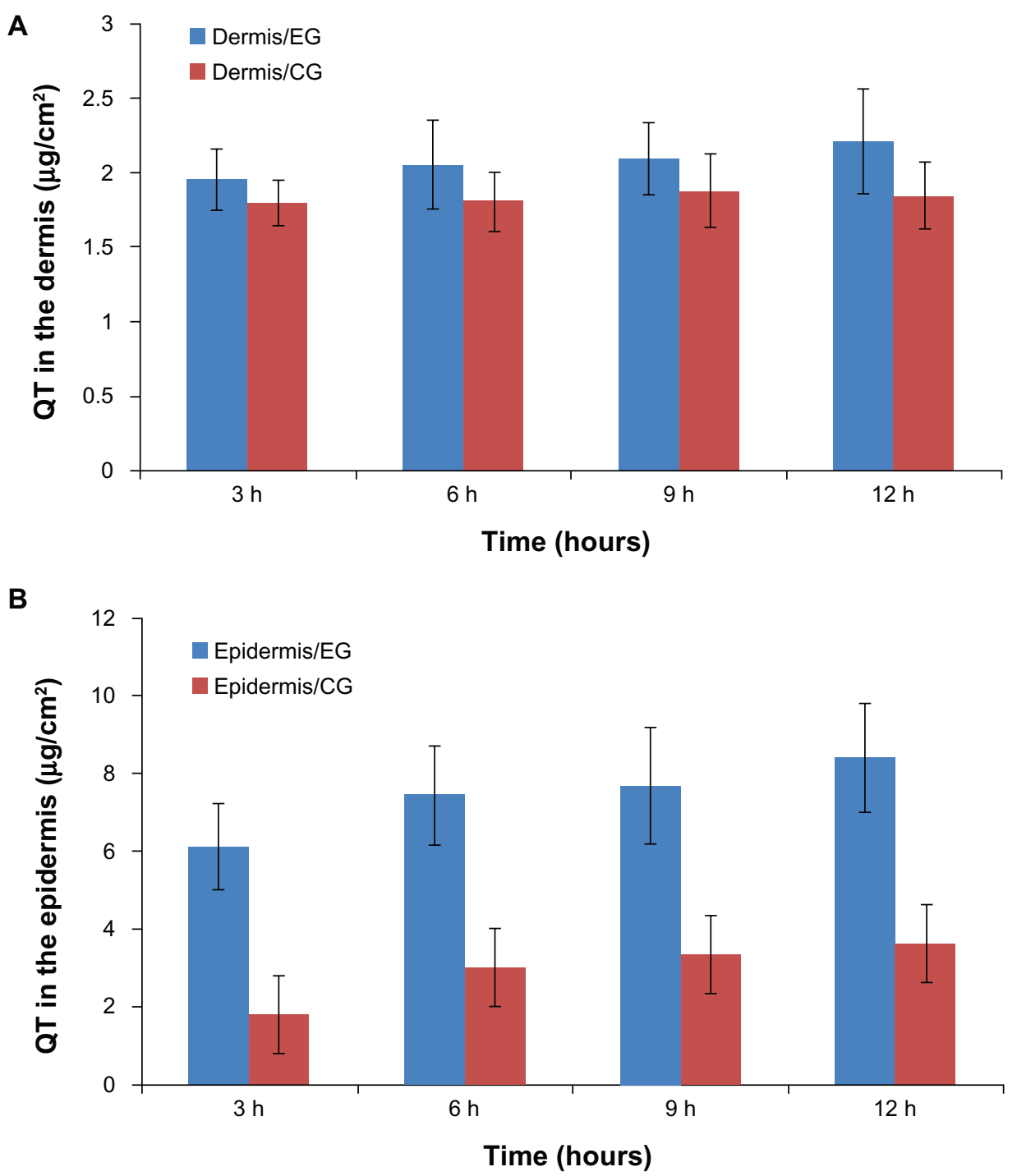

Figure 6 Amount of quercetin in the (A) dermis and (B) epidermis after hours 3, 6, 9, and 12, respectively.

Note: Results are represented by means \pm standard deviation $(n=3)$.

Abbreviations: CG, control group; EG, experimental group.

furthermore TPGS could also increase the diffusion coefficient of the drug between the skin and the vehicle. ${ }^{46,56,57}$

To sum up, the results demonstrate that, compared with the control formulation, nanoparticles could significantly promote the accumulation of quercetin in skin, especially in the epidermis. Because the efficacy of quercetin in delaying ultraviolet radiation-mediated oxidant injury and cell death mainly occurs in the epidermis, it is of great significance for the nanoparticles to make quercetin remain in skin, especially in the epidermis.

\section{Effect of nanoparticles on the surface of mice skin}

Microscopic pictures of untreated skin, skin treated with quercetin propylene glycol solution, and quercetin-loaded nanoparticles are shown in Figure 7. Untreated skin (Figure 7A) had a compact stratum corneum, with the corneocyte layer closely conjugated. Application of quercetin propylene glycol solution could slightly change the tight structure of the stratum corneum (Figure 7B). The skin treated with the nanoparticle suspension appeared swollen, with increased overall thickness of the stratum corneum and, furthermore, loose cell junctions and increased intercellular space could also be observed (Figure 7C). The change in the skin surface resulting from the effect of the nanoparticles might be related to their composition. The positive charge of chitosan ensured close contact between the nanoparticles and the skin. The hydrated outer environment provided by chitosan and the active surface nature of TPGS provided better wetting to the skin, which might significantly alter the 

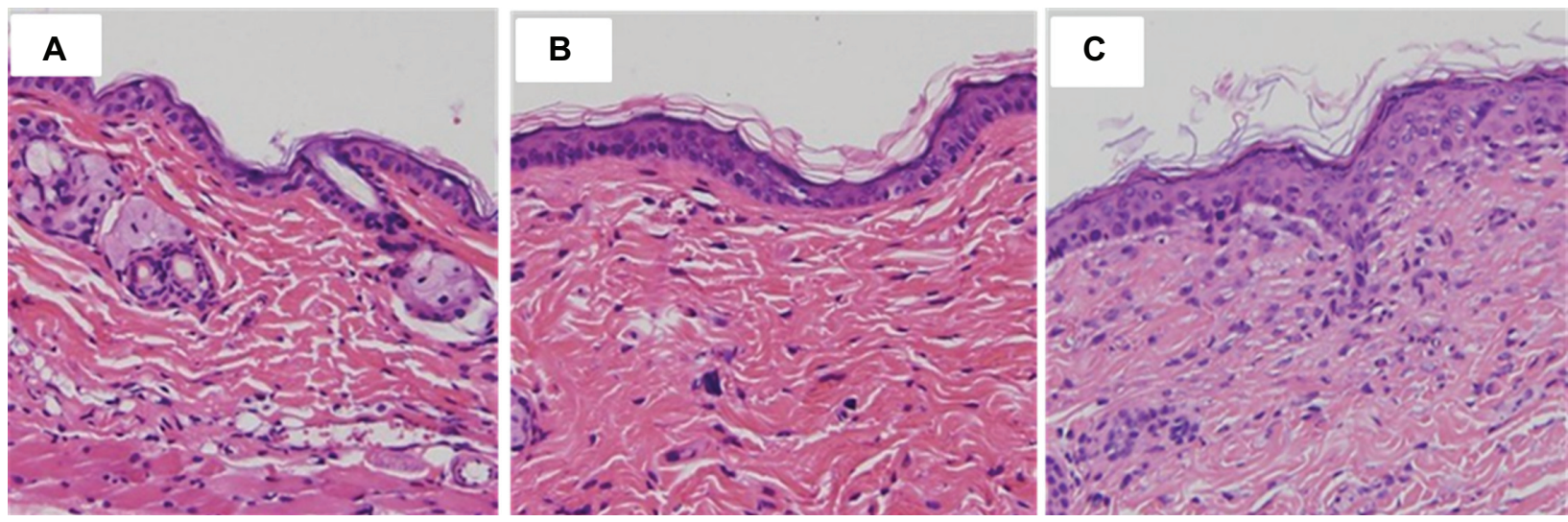

Figure 7 Microphotograph $(200 \times)$ of vertical section of mouse skin after hematoxylin eosin staining: (A) untreated skin, (B) skin treated with quercetin propylene glycol solution, and $(\mathbf{C})$ skin treated with quercetin-loaded NPs.

barrier properties and reduce or prevent skin dehydration. Also, increased hydration of the stratum corneum could facilitate permeation of drug into the epidermis. Following interaction between the skin and the nanoparticles, the exposed lecithin could mix and fuse with lipids in the skin to loosen the structure of the skin and disturb the lamellar arrangement of the lipids to increase the thickness of the stratum corneum. ${ }^{38,58}$ The effects of the nanoparticles and their ingredients, including chitosan, TPGS, and lecithin, on the skin weakened the stratum corneum barrier and facilitated drug permeation.

\section{Conclusion}

Quercetin-loaded chitosan-lecithin nanoparticles were prepared in this study for topical administration. Addition of TPGS into the nanosystem resulted in increased entrapment efficiency and drug-loading of quercetin. Compared with the quercetin propylene glycol solution, the quercetin-loaded nanoparticles could notably enhance the skin permeation of quercetin in vitro and in vivo, and effectively promote the retention of quercetin in the epidermis. The interaction between nanoparticles and the skin surface changed the morphology of the stratum corneum and broke the close conjugation of the corneocyte layers, which resulted in the increased permeation of quercetin into the skin. It can be concluded that the obtained formulation of chitosanlecithin nanoparticles could be a promising vehicle for topical delivery of quercetin.

\section{Acknowledgment}

This work was supported by a research grant from the Science and Technology Department of Shandong University, People's Republic of China.

\section{Disclosure}

The authors report no conflicts of interest in this work.

\section{References}

1. Saijaa A, Tomainoa A, Trombettaa D, Giacchib M, Pasqualea AD, Bonina F. Influence of different penetration enhancers on in vitro skin permeation and in vivo photoprotective effect of flavonoids. Int J Pharm. 1998;175(1):85-94.

2. Skaper SD, Fabris M, Ferrari V, Carbonar MD, Leon A. Quercetin protects cutaneous tissue-associated cell types including sensory neurons from oxidative stress induced by glutathione depletion: cooperative effects of ascorbic acid. Free Radic Biol Med. 1996;22(4):669-678.

3. Wiseman H, Halliwell B. Damage to DNA by reactive oxygen and nitrogen species: role in inflammatory disease and progression to cancer. Biochem J. 1996;313(1):17-29.

4. Bors W, Heller W, Michel C, Saran M. Flavonoids as antioxidants: determination of radical-scavenging efficiencies. Methods Enzymol. 1990; $186: 34355$.

5. Bors W, Heller W, Michel C, Saran M. Flavonoid antioxidants: rate constants for reactions with oxygen radicals. Methods Enzymol. 1994;234:420-429.

6. Laughton MJ, Evans PJ, Moroney MA, Hoult JRS, Halliwell B. Inhibition of mammalian 5-lipoxygenase and cyclo-oxygenase by flavonoids and phenolic dietary additives. Relationship to antioxidant activity and to iron ion-reducing ability. Biochem Pharmacol. 1991; 42(9):1673-1681.

7. Decharneux T, Dubois F, Beauloye C, Wattiaux-De Coninck S, Wattiaux R. Effect of various flavonoids on lysosomes subjected to an oxidative stress. Biochem Pharmacol. 1992;44(7):1243-1248.

8. Torel J, Cillard J, Cillard P. Antioxidant activity of flavonoids and reactivity with peroxy radical. Phytochemistry. 1986;25(2):383-386.

9. Cao G, Sofic E, Prior RL. Antioxidant and prooxidant behavior of flavonoids: structure-activity relationships. Free Radic Biol Med. 1997;22(5):749-760.

10. Kumari A, Yadav SK, Pakade YB, Singh B, Yadav SC. Development of biodegradable nanoparticles for delivery of quercetin. Colloids Surf B Biointerfaces. 2010;80(2):184-192.

11. Choksi AN, Poonawalla T, Wilkerson MG. Nanoparticles: a closer look at their dermal effects. J Drugs Dermatol. 2010;9(5):475-481.

12. Gamer AO, Leibold E, van Ravenzwaay B. The in vitro absorption of microfine zinc oxide and titanium dioxide through porcine skin. Toxicol in Vitro. 2006;20(3):301-307.

13. Cross SE, Innes B, Roberts MS, Tsuzuki T, Robertson TA, McCormick P. Human skin penetration of sunscreen NPs: in-vitro assessment of a novel micronized zinc oxide formulation. Skin Pharmacol Physiol. 2007;20(3):148-154. 
14. Kelmanna RG, Kumineka G, Teixeirab HF, Koestera LS. Carbamazepine parenteral nanoemulsions prepared by spontaneous emulsification process. Int J Pharm. 2007;342(1-2):231-239.

15. Pavelić Z, Skalko-Basnet N, Filipović-Grcić J, Martinac A, Jalsenjak I. Development and in vitro evaluation of a liposomal vaginal delivery system for acyclovir. $J$ Control Release. 2005;106(1-2): 34-43.

16. Ceraulo L, Fanara S, Turco Liveri V, Ruggirello A, Panzeri W, Mele A. Orientation and molecular contacts of melatonin confined into AOT and lecithin reversed micellar systems. Colloids Surf A Physicochem Eng Asp. 2008;316(1-3):307-312.

17. Schubert MA, Harms M, Müller-Goymann CC. Structural investigations on lipid NPs containing high amounts of lecithin. Eur J Pharm Sci. 2006;27(2-3):226-236.

18. Sonvico F, Cagnani A, Rossi A, et al. Formation of self-organized NPs by lecithin/chitosan ionic interaction. Int J Pharm. 2006;324(1): 67-73.

19. Şenyiğit T, Sonvico F, Barbieri S, Özer Ö, Santi P, Colombo P. Lecithin/ chitosan NPs of clobetasol-17-propionate capable of accumulation in pig skin. J Control Release. 2010;142(3):368-373.

20. Liu HT, Li WM, Xu G, et al. Chitosan oligosaccharides attenuate hydrogen peroxide-induced stress injury in human umbilical vein endothelial cells. Pharmacol Res. 2009;59(3):167-175.

21. Park PJ, Je JY, Kim SK. Free radical scavenging activity of chito oligosaccharides by electron spin resonance spectrometry. J Agric Food Chem. 2003;51(16):4624-4627.

22. Yoon HJ, Moon ME, Park HS, Im SY, Kim YH. Chitosan oligosaccharide (COS) inhibits LPS-induced inflammatory effects in RAW 264.7 macrophage cells. Biochem Biophys Res Commun. 2007;358(3): 954-959.

23. Qiao Y, Bai XF, Du YG. Chitosan oligosaccharides protect mice from LPS challenge by attenuation of inflammation and oxidative stress. Int Immunopharmacol. 2011;11(1):121-127.

24. Fakhry A, Schneider GB, Zaharias R, Senel S. Chitosan supports the initial attachment and spreading of osteoblasts preferentially over fibroblasts. Biomaterials. 2004;25(11):2075-2079.

25. Singla AK, Chawla M. Chitosan: some pharmaceutical and biological aspects - an update. J Pharm Pharmacol. 2001;53(8):1047-1067.

26. Illum L. Chitosan and its use as a pharmaceutical excipient. Pharm Res. 1998;15(9):1326-1331.

27. Agnihotri SA, Mallikarjuna NN, Aminabhavi TM. Recent advances on chitosan-based micro- and NPs in drug delivery. J Control Release. 2004;100(1):5-28

28. Sung HW, Chen MC, Lin YH, Liang HF, Tu H, inventors. GP Medical Inc, National Tsing Hua University, Assignee. Nanoparticles for protein drug delivery. US Patent 7919293. April 5, 2011.

29. Hammes M, Singh A. Effect of polycations on permeability of glomerular epithelial cell monolayers to albumin. J Lab Clin Med. 1994;123(3):437-446.

30. Mei D, Mao S, Sun W, Wang Y, Kissel T. Effect of chitosan structure properties and molecular weight on the intranasal absorption of tetramethylpyrazine phosphate in rats. Eur J Pharm Biopharm. 2008; 70(3):874-881.

31. Langoth N, Kahlbacher H, Schöffmann G, et al. Thiolated chitosans: design and in vivo evaluation of a mucoadhesive buccal peptide drug delivery system. Pharm Res. 2006;23(3):573-579.

32. Schipper NG, Olsson S, Hoogstraate JA, deBoer AG, Vårum KM, Artursson P. Chitosans as absorption enhancers for poorly absorbable drugs. 2: mechanism of absorption enhancement. Pharm Res. 1997; 14(7):923-929.

33. Ozcan I, Abaci O, Uztan AH, et al. Enhanced topical delivery of terbinafine hydrochloride with chitosan hydrogels. AAPS Pharm Sci Tech. 2009;10(3):1024-1031.

34. Wu X, Landfester K, Musyanovych A, Guy RH. Disposition of charged nanoparticles after their topical application to the skin. Skin Pharmacol Physiol. 2010;23(3):117-123.
35. Zhu W, Yu A, Wang W, Dong R, Wu J, Zhai G. Formulation design of microemulsion for dermal delivery of penciclovir. Int J Pharm. 2008; 360(1-2):184-190.

36. Sorensen EN, Weisman G, Vidaver GA. A Sephadex column procedure for measuring uptake and loss of low molecular weight solutes from small, lipid-rich vesicles. Anal Biochem. 1977;82(2):376-384.

37. Singh B, Mehta G, Kumar R, Bhatia A, Ahuja N, Katare OP. Design, development and optimization of nimesulide-loaded liposomal systems for topical application. Curr Drug Deliv. 2005;2(2):143-153.

38. Lv Q, Yu A, Xi Y, et al. Development and evaluation of penciclovirloaded solid lipid NPs for topical delivery. Int J Pharm. 2009;372(1-2): 191-198.

39. Zhao X, Liu JP, Zhang X, Li Y. Enhancement of transdermal delivery of theophylline using microemulsion vehicle. Int $J$ Pharm. 2006;327(1-2):58-64.

40. Puglia C, Blasi P, Rizza L, et al. Lipid NPs for prolonged topical delivery: An in vitro and in vivo investigation. Int J Pharm. 2008; 357(1-2): 295-304.

41. Fang JY, Fang CL, Liu CH, Su YH. Lipid NPs as vehicles for topical psoralen delivery: solid lipid nanoparticles (SLN) versus nanostructured lipid carriers (NLC). Eur J Pharm Biopharm. 2008;70(2): 633-640.

42. Mu L, Seow PH. Application of TPGS in polymeric nanoparticulate drug delivery system. Colloids Surf B Biointerfaces. 2006;47(1): 90-97.

43. Gao Y, Li LB, Zhai G. Preparation and characterization of Pluronic/ TPGS mixed micelles for solubilization of camptothecin. Colloids Surf B Biointerfaces. 2008;64(2):194-199.

44. Gao L, Zhang D, Chen M, et al. Studies on pharmacokinetics and tissue distribution of oridonin nanosuspensions. Int J Pharm. 2008; 355(1-2):321-327.

45. Repka MA, McGinity JW. Bioadhesive properties of hydroxypropylcellulose topical films produced by hot-melt extrusion. J Control Release. 2001;70(3):341-351.

46. Chang T, Benet LZ, Hebert MF. The effect of water-soluble vitamin E on cyclosporine pharmacokinetics in healthy volunteers. Clin Pharmacol Ther. 1996;59(3):297-303.

47. Yuan H, Lu LJ, Du YZ, Hu FQ. Stearic acid-g-chitosan polymeric micelle for oral drug delivery: in vitro transport and in vivo absorption. Mol Pharm. 2011;8(1):2252-2238.

48. Liu W, Guo Y. Interaction between flavonoid, quercetin and surfactant aggregates with different charges. J Colloid Interface Sci. 2006;302(2): 625-632.

49. Mishra PR, Al Shaal L, Müller RH, Keck CM. Production and characterization of hesperetin nanosuspensions for dermal delivery. Int J Pharm. 2009;371(1-2):182-189.

50. Van Eerdenbrugh B, Van den Mooter G, Augustijns P. Top-down production of drug nanocrystals: nanosuspension stabilization, miniaturization and transformation into solid products. Int $J$ Pharm. 2008;364(1):64-75.

51. Baert L, van't Klooster G, Dries W, et al. Development of a long-acting injectable formulation with nanoparticles of rilpivirine (TMC278) for HIV treatment. Eur J Pharm Biopharm. 2009;72(3):502-508.

52. Liu J, Hu W, Chen H, Ni Q, Xu H, Yang X. Isotretinoin-loaded solid lipid nanoparticles with skin targeting for topical delivery. Int J Pharm. 2007;328(2):191-195

53. Chen H, Chang X, Du D, et al. Podophyllotoxin-loaded solid lipid nanoparticles for epidermal targeting. J Control Release. 2006;110(2): 296-306.

54. Lombardi Borgia S, Regehly M, Sivaramakrishnan R, et al. Lipid nanoparticles for skin penetration enhancement-correlation to drug localization within the particle matrix as determined by fluorescence and parelectric spectroscopy. J Control Release. 2005;110(1):151-163.

55. Reifenrath WG, Hawkins GS, Kurtz MS. Percutaneous penetration and skin retention of topically applied compounds: an in vitro-in vivo study. J Pharm Sci. 1991;80(6):526-532. 
56. Zhu W, Guo C, Yua A, Gao Y, Cao F, Zhai G. Microemulsion-based hydrogel formulation of penciclovir for topical delivery. Int J Pharm. 2009;378(1-2):152-158.

57. El Maghraby GM. Transdermal delivery of hydrocortisone from eucalyptus oil microemulsion: effects of cosurfactants. Int J Pharm. 2008;355(1-2):285-292.
58. El Maghraby GM, Barry BW, Williams AC. Liposomes and skin: from drug delivery to model membranes. Eur J Pharm Sci. 2008;34(4-5): 203-222.

\section{Publish your work in this journal}

The International Journal of Nanomedicine is an international, peerreviewed journal focusing on the application of nanotechnology in diagnostics, therapeutics, and drug delivery systems throughout the biomedical field. This journal is indexed on PubMed Central, MedLine, CAS, SciSearch $\AA$, Current Contents ${ }^{\circledR} /$ Clinical Medicine,
Journal Citation Reports/Science Edition, EMBase, Scopus and the Elsevier Bibliographic databases. The manuscript management system is completely online and includes a very quick and fair peer-review system, which is all easy to use. Visit http://www.dovepress.com/ testimonials.php to read real quotes from published authors. 